\title{
High-energy lateral mapping (HELM) studies of inhomogeneity and failure mechanisms in NMC622/Li pouch cells
}

Gerard S. Mattei §,॥, Zhuo Li§,॥, Adam A. Corrao§,", Chaojiang Niu†, Yulun Zhang\#, Boryann Liaw", Charles C. Dickerson\#, Jie Xiao ${ }^{\dagger, \&}$, Eric J. Dufek\#, and Peter G. Khalifah", ${ }^{\S, *}$

$\S$ Department of Chemistry, Stony Brook University, Stony Brook, New York, Untied States 11794

" Chemistry Division, Brookhaven National Laboratory, Upton, New York, United States 11973

† Energy and Environmental Directorate, Pacific Northwest National Laboratory, Richland, Washington, United States 99352

\# Energy Storage and Advanced Transportation Department, Idaho National Laboratory, Idaho Falls, ID, United States 83415

${ }^{\&}$ Materials Science and Engineering Department, University of Washington, Seattle, WA, United States 98195 


\section{High Energy Lateral Mapping (HELM)}

\section{Synchrotron XRD mapping}

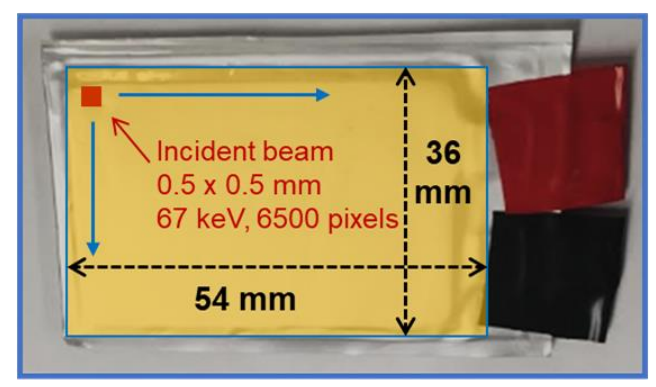

Automated Rietveld refinement of XRD data

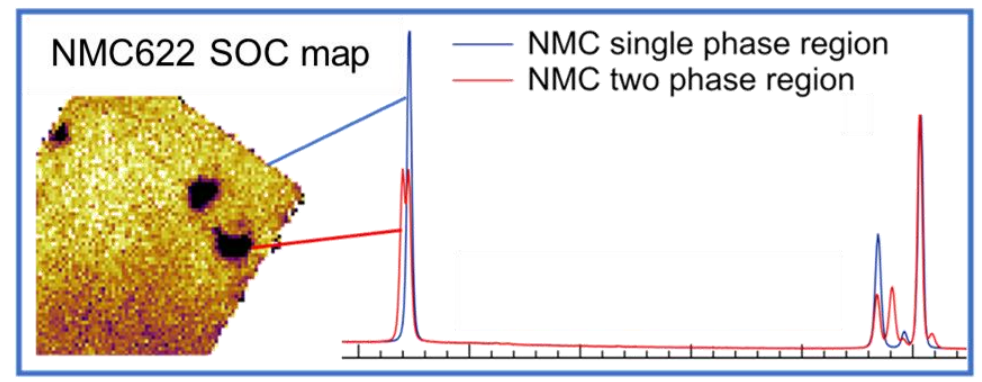

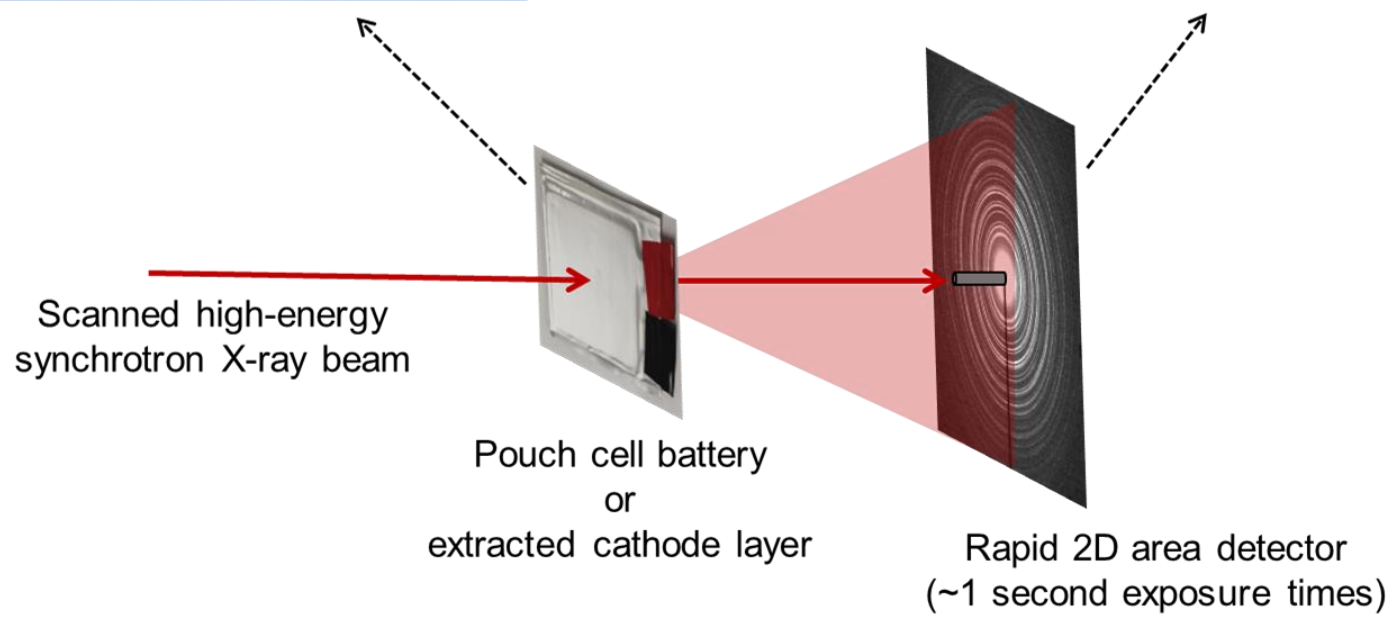

Figure S1. Schematic of the high-energy lateral mapping (HELM) approach. A small synchrotron X-ray beam $(0.5 \times 0.5 \mathrm{~mm})$ is used to scan across the sample, consisting of either a whole battery cell or a single extracted electrode layer (top left image). The combination of an intense synchrotron beam and a large area detector enables high quality powder diffraction data to be rapidly collected ( 1 sec / pattern). This permits a $2 \mathrm{D}$ array of diffraction patterns to be collect in an hour or two for industrially relevant pouch cell battery samples. The subsequent automated Rietveld analysis of the X-ray diffraction data is used to determine the battery state of charge (SOC) for each pixel on the map, allowing the phase behavior to be quantified and battery cell inhomogeneity and failure mechanisms to be probed. 

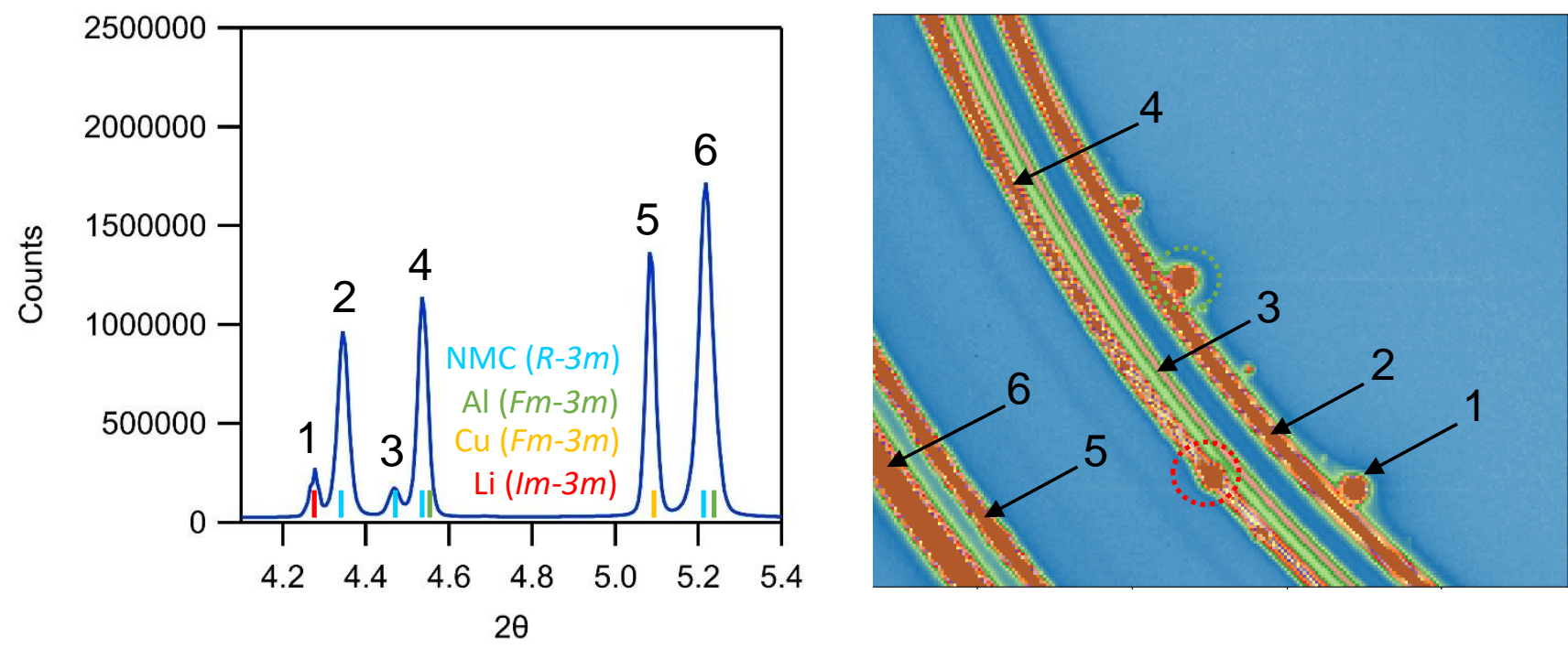

Figure S2. Zoomed in region of a representative 1D diffraction pattern (left) and corresponding 2D image (right) collected at the center of the pristine 7-layer pouch cell, with numbers used to indicate the correspondence between peaks in the 1D pattern and rings in the 2D pattern. Colored tick marks in 1D pattern indicate which phases contribute to which diffraction peaks. Significant spottiness in rings can be clearly seen for the Li phase in ring \#1 (example of a spot indicated with a red circle) and for the Al phase in ring \#4 (green circle), indicative of the large grain sizes for these two phases. 


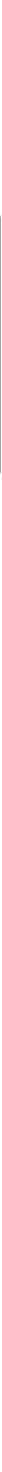

Figure S3. Pixel map of relative phase abundances of NMC622 in a pristine 7-layer pouch cell, as was previously shown in Figure 3 but with the addition of labels for the phase abundance on each pixel. 

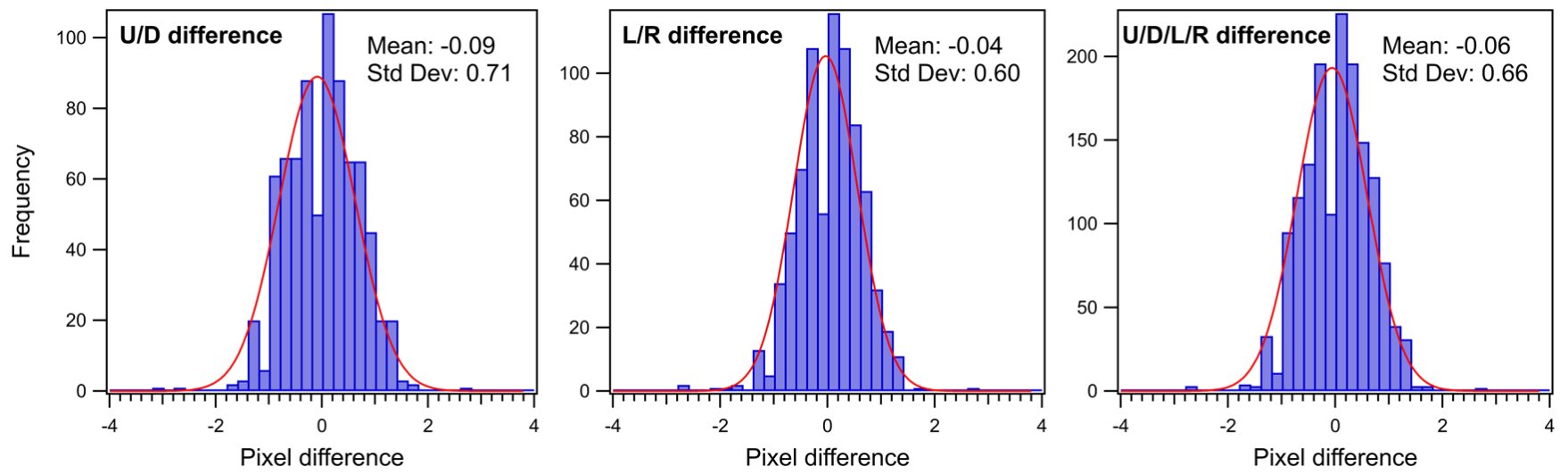

Figure S4. Histograms showing the difference in phase abundance pixel value for pixels above/below the current pixel (top), left/right (middle), and combined (bottom).
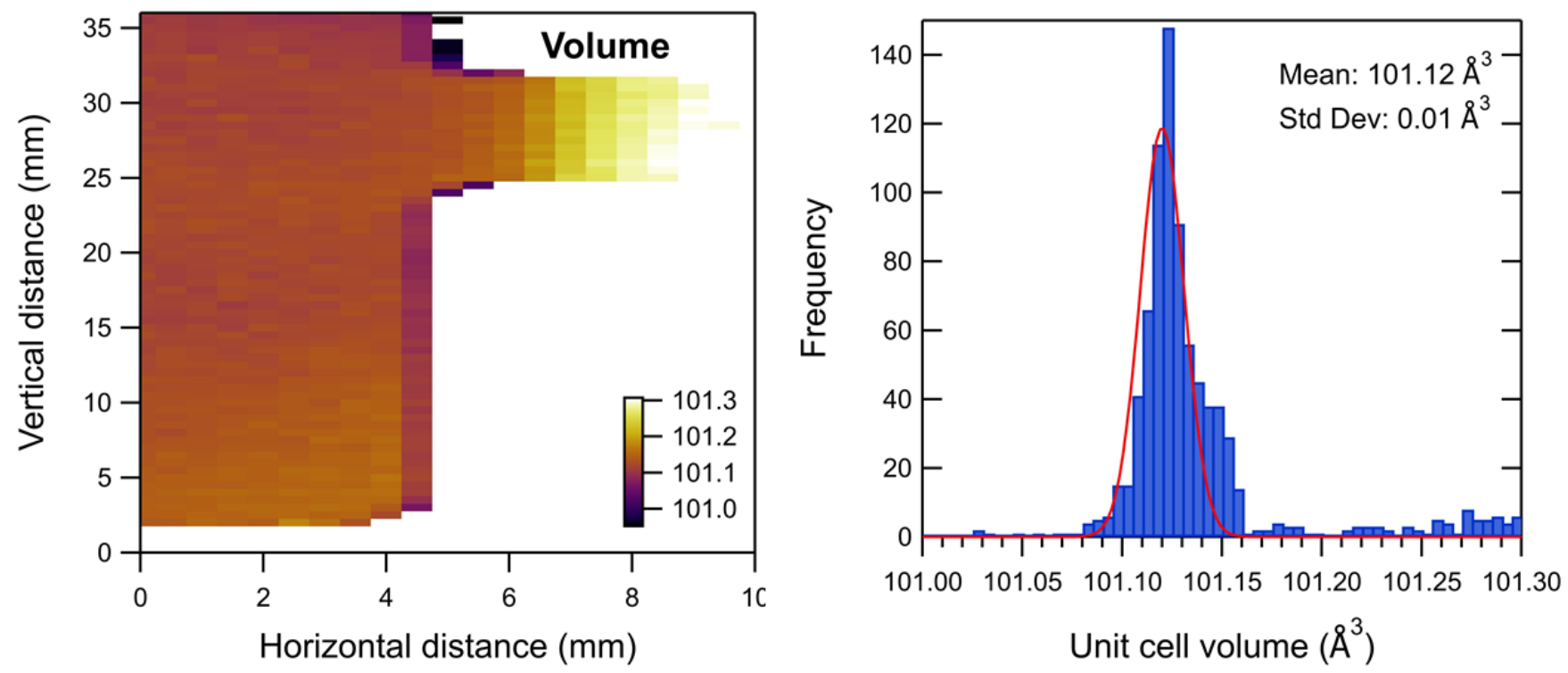

Figure S5. Unit cell volume map (left) and histogram (right) of pristine NMC in a 7-layer pouch cell prior to correction using $\mathrm{Al}$ metal as an internal standard. 

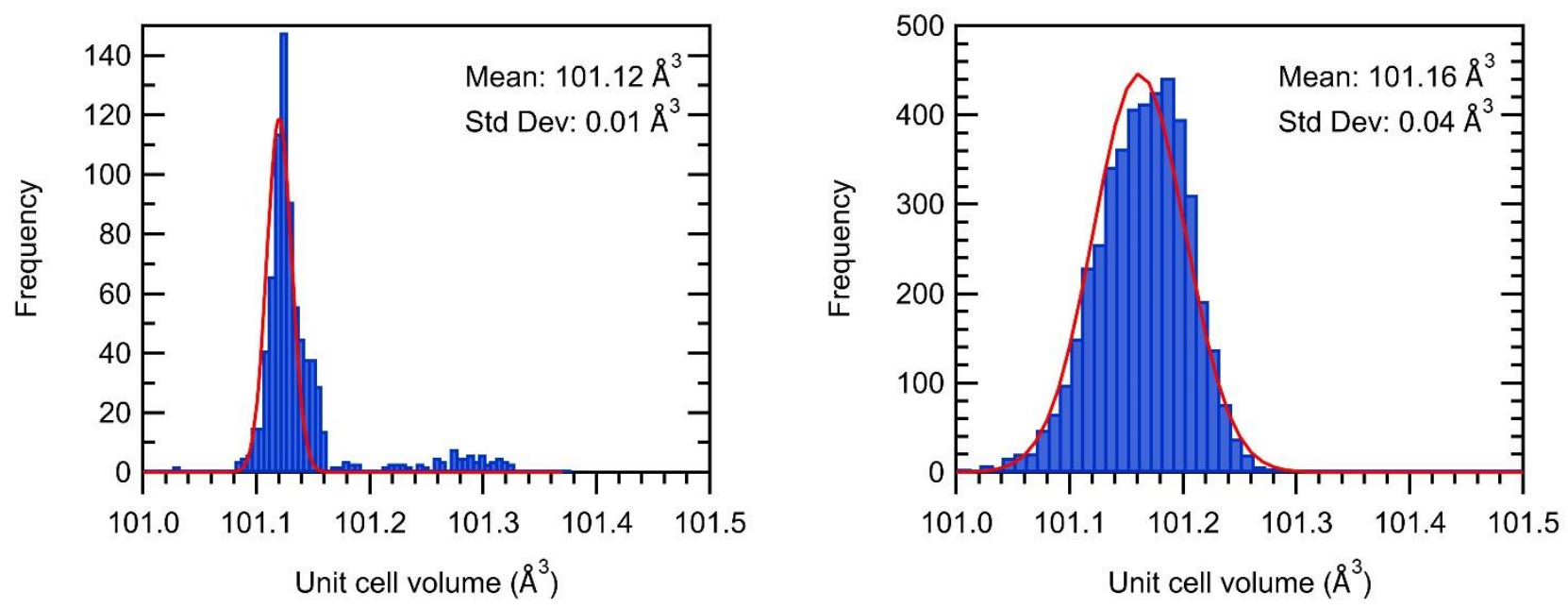

Figure S6. Unit cell volume histograms for NMC622 in 7-layer pouch cell (left) and cycled single-layer film (right). For regions where 2-phase NMC622 is present in the cycled film, the unit cell volume is shown for phase A, which exhibits similar cell volumes to that of the pristine cell.
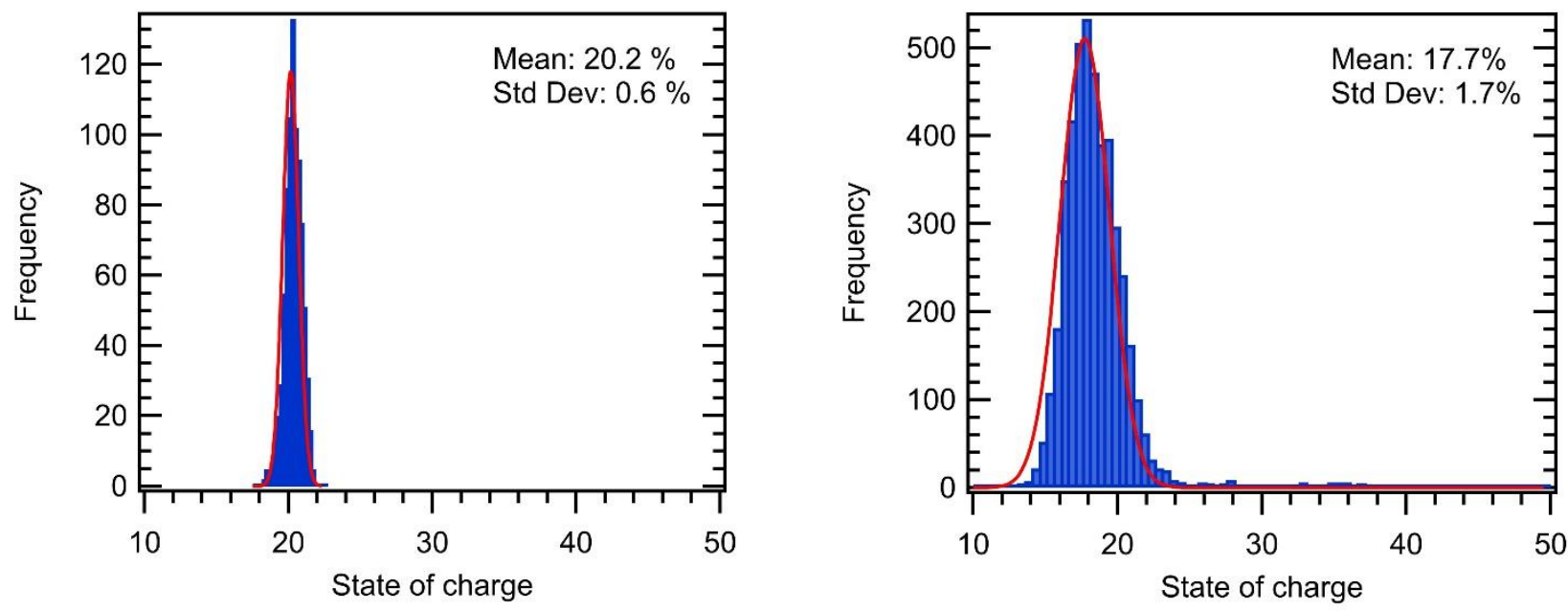

Figure S7. State of charge histograms for NMC622 in 7-layer pouch cell (left) and cycled single-layer film (right). For regions where 2-phase NMC622 is present in the cycled film, state of charge is calculated as the sum of the SOC of each phase normalized to their respective phase fractions. 

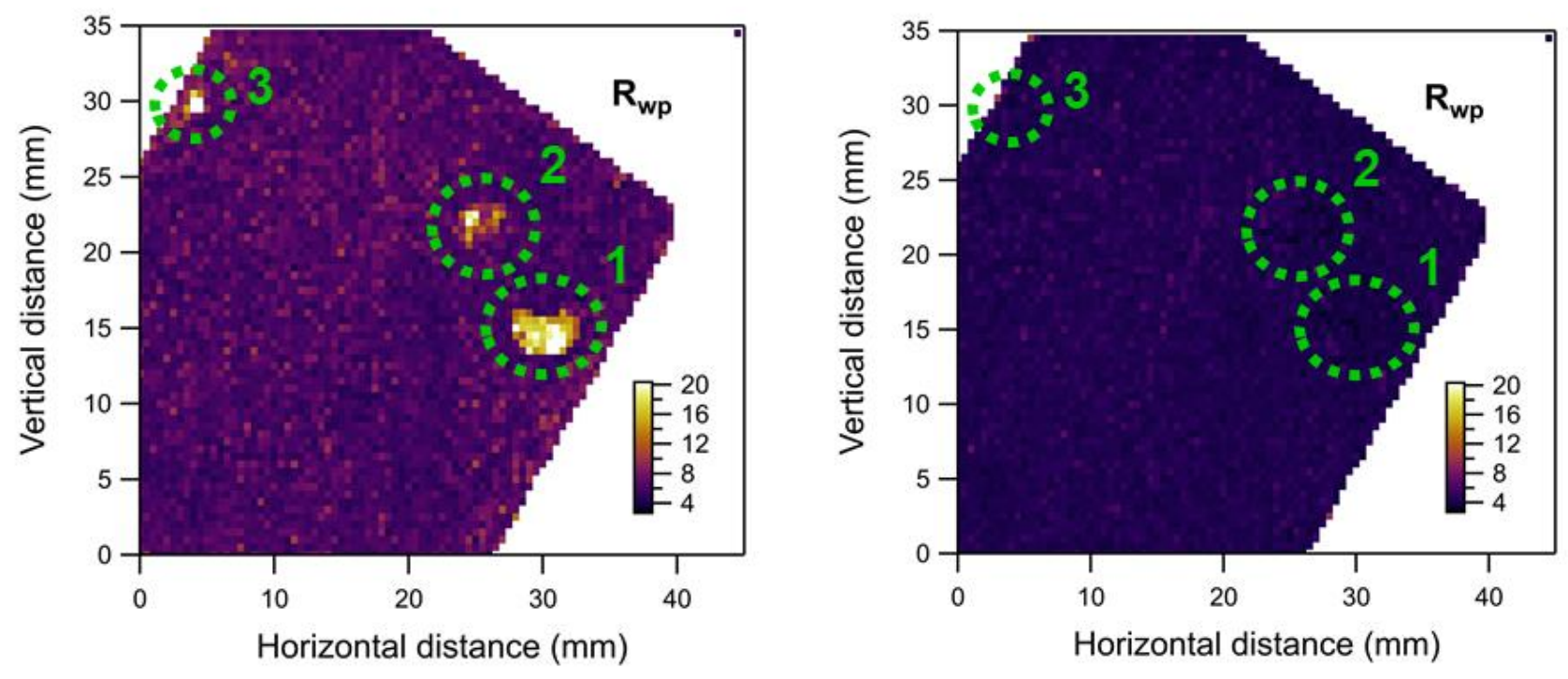

Figure S8. Maps of the $R_{\mathrm{wp}}$ for Rietveld refinements using a single NMC phase (left) and two separate NMC phases each with a different SOC (right). It can be clearly seen that that the three hot spots cannot be effectively modeled using a single NMC phase, consistent with the peak splitting directly observed in $\mathrm{X}$-ray diffraction patterns for pixels in these regions (Figure 7). Note that in the two phase refinements, it was observed that one of the two NMC phases has a SOC that is low and is indistinguishable from the surrounding regions ("A" phase), while the other is substantially higher and is generally consistent within the spot ("B" phase). 